\title{
Research on Evaluation of Network Planning Based on Rough Set
}

\author{
$\mathrm{Xu} \mathrm{ZHENG}^{*}$ \\ Economic and Technical Research Institute of State \\ Grid Hubei Power Company \\ Wuhan, P.R.China \\ e-mail: 20688068@qq.com \\ Xiu-wen XIONG \\ Economic and Technical Research Institute of State Grid \\ Hubei Power Company \\ Wuhan, P.R.China \\ Kai YUAN \\ School of Information Engineering, Nanchang University \\ Nanchang, P.R.China \\ e-mail: 1946275214@qq.com
}

\author{
Yong HUANG \\ Economic and Technical Research Institute of State \\ Grid Hubei Power Company \\ Wuhan, P.R.China
}

\author{
Da-wei HUANG \\ Economic and Technical Research Institute of State Grid \\ Hubei Power Company \\ Wuhan, P.R.China
}

\author{
Min XU \\ School of Information Engineering, Nanchang University \\ Nanchang, P.R.China
}

\begin{abstract}
An evaluation method of network planning based on rough set theory is proposed. Index weights assigning method is used to evaluate the coordination of network planning with the indexes of power system in reliability, safety, economy and environmental impact. On the principle of attribute importance of rough set theory to determine the relationship between each evaluation index. Index weights determined entirely by the law of the data, it avoid subjective arbitrariness of subjective weighting method and uncertainty disadvantages of objective weighting method, so as to obtain more reliability and accuracy in evaluation. It is proved to be effectiveness and feasibility of the proposed method in the case of comprehensive evaluation in network planning.
\end{abstract}

Keywords- Network Planning; Evaluation; Rough
Set;Weights

\section{INTRODUCTION}

With the deepening of the power system reform [1], the the power grids planning have been affected by more and more uncertain factors. The previous grid planning evaluation methods have been difficult to apply. The coordinated development of the power grid relates the safe operation and economic operation of the power grid, and the multi-level coordinated development between the three parties(Power supply, grid, load) and the external environment will also determine the prospects of sustainable development of the power grid. Therefore, it is important to evaluate the coordination degree of the power grid planning[2]. However, due to the various indexes involved in the various aspects of grid planning, it is difficult to evaluate the coordination of power grid planning and the objectivity of evalution of the power grid planning[3].Index weighting method applicated more in the multi-indexes comprehensive evaluation[4], and there are various types of problems in previous indexes weighting evaluation method, especially in the determine of index weight. The indexes weighting method can be divided into subjective weighting method and objective weighting method [5]. Among them, the index weight determined by subjective weighting method is more consistent with the actual situation, but it is affected more by subjective assumption in the prosess of empowerment, it will affect the objectivity of the evaluation results [6].

Rough set theory as a mathematical method to deal with uncertain and incomplete knowledge [7-8], it performs data mining around a given data set in data processing without another irrelevant knowledge of research, analyzes the hidden rules of data, explores the interrelationships of data according to the problem researched, and determines the importance of the various types of data researched based on previous empirical data and the interrelationships between the data explored [6]. In this paper, the principle of attribute importance in the rough set theory is used to evaluate the coordination of power network planning in order to avoid the shortcomings of the previous indicator weighting methods.

\section{BASIC CONCEPTS OF ROUGH SET}

\section{A. Knowledge Representation}

Array $\mathrm{S}=(U, A, \quad V, f)$ constitute a knowledge representation system, $\mathrm{U}$ is called a nonempty finite set of objects, also known as the domain; $\mathrm{A}$ is a nonempty finite set of attributes; $V=\bigcup_{a \in A} V_{a}$, Va is the range of the attribute a; f: $U \times A \rightarrow V$ is a information function [9]. A knowledge representation system is also called an information system, 
the attributes set $A=C \cup D, C \cap D \neq \varnothing, \mathrm{C}$ is the condition attribute set, $\mathrm{D}$ is the decision attribute set. If the attribute set of knowledge system includes condition attribute and decision attribute, it is called decision table.

\section{B. Knowledge Dependency}

Neighborhood decision system $S=(U, C \cup D), C$ is the condition attribute set, $D$ is the decision attribute set. According to the decision attribute $D$, the domain $U$ is divided into $\mathrm{N}$ equivalence classes: $X_{1}, X_{2}, \ldots, X_{\mathrm{N}}$, then the upper approximation and the lower approximation of decision attribute $D$ with respect to conditional attribute $C$ is defined as:

$$
\begin{aligned}
& \frac{N_{C} D}{} D=\bigcup_{i=1}^{N} \underline{N_{C}} X_{i} \\
& \overline{N_{C}} D=\bigcup_{i=1}^{N} \overline{N_{C}} X_{i}
\end{aligned}
$$

The lower approximation is also called the positive domain, denoted as $P_{\mathrm{C}}(D)$. The dependency of decision attribute $D$ on conditional attribute $C$ is defined as:

$$
k=\gamma_{C}(D)=\frac{\operatorname{Card}\left(P_{C}(D)\right)}{\operatorname{Card}(U)}
$$

Which $0 \leq k \leq 1$, The dependency of decision attribute $D$ on conditional attribute $C-\left\{c_{j}\right\}$ is defined as:

$$
\gamma_{C-\left\{c_{j}\right\}}(D)=\frac{\left.\operatorname{Card}\left(P_{C-\left\{c_{j}\right\}}\right\}(D)\right)}{\operatorname{Card}(U)}
$$

\section{Attribute Importance}

In the neighborhood decision system $S=(U, C \cup D, V, f), \forall c \in C$, the importance of the condition attribute ${ }^{c_{j}}$ to decision attribute $\mathrm{D}$ is defined as:

$$
Z\left(c_{j}, C, D\right)=\gamma_{C}(D)-\gamma_{C-\left\{c_{j}\right\}}(D)
$$

The higher the value of $Z\left(c_{j}, C, D\right)$, the greater the importance of the condition attribute; the other hand, the lower the importance of the attribute.

\section{Rough Set Evaluation Model}

The evaluation method of multi-index system by rough set theory is mainly to associate the evaluation indicator with the attribute of rough set to determine the importance of the attribute to determine the weight of index. Through the mining of the index data and the analysis of the importance of the evaluation object, the weight coefficient value of the multi-index system is determined.

\section{A. Determination of Attributes}

The evaluation index of each evaluation object is taken as the condition attribute, and the condition attribute set $C=\left\{c_{1}, c_{2}, \cdots, c_{n}\right\}$ can be obtained; the decision attribute is the score of each index, and the decision attribute set $D=\{y\}$ is obtained; then the decision table is created. The evaluation object is the row of the decision table, and the evaluation indicator is the column of the decision table. The condition attribute value ci and decision attribute value yk of the object to be evaluated are taken as a piece of information of the knowledge system, and $u_{k}=\left\{c_{1 k}, c_{2 k}, \cdots, c_{n k}, y_{k}\right\}_{\text {can }}$ be obtained, then $U=\left\{u_{1}, u_{2}, \cdots, u_{m}\right\}_{\text {is }}$ the domain which is the decision table of multi-index evaluation system.

\section{B. Processing of Attribute Data}

In the evaluation index system, The attribute value y of decision attribute $\mathrm{D}$ can be obtained by condition attribute $\mathrm{C}$. First, each conditional attribute value cij of each evaluation object is scored to obtain the fractional yij of cij, and the decision attribute value $y$ of the evaluation object is obtained by averaging and discretizing yij. In the aspect of index scoring, the normalized method is adopted to eliminate the difference of the magnitude and dimension of each index data, As the specific requirements of the different indexes, the greater the value of some indexes the higher the score, the smaller the value of some indexes the higher the score[6]. The scoring method is as follows:

When the evaluation index value is smaller the higher the score,

$$
y_{i j}=\frac{c_{j \max }-c_{i j}}{c_{j \max }-c_{j \min }} \times 100 \%
$$
score

When the evaluation index value is greater the higher the

$$
y_{i j}=\frac{c_{i j}-c_{j \min }}{c_{j \max }-c_{j \min }} \times 100 \%
$$

\section{Determination of Attribute Weights and Evaluation Results}

The index weights of multi-index evaluation system can be determined by the principle of attribute importance in rough sets. From the rough set theory, the knowledge system will change its classification condition because of the addition of some attributes, but it will not change the classification condition because of the removal of some attributes. In order to get the importance of the attribute, we first remove the attribute, and then compare the change degree of knowledge system classification. If the 
classification of the knowledge system changes greatly, it indicates that the importance of the attribute is high; conversely, the importance of the attribute is low. Proceed as follows:

(1)Determine the set of equivalence classes: $U / \operatorname{ind}(C)$, $U / \operatorname{ind}(D), U / \operatorname{ind}\left(C-\left\{c_{j}\right\}\right)$;

(2)Determine the condition attribute positive domain: $P_{C}(D), P_{C-\left\{c_{j}\right\}}(D)$;

(3)Determine the cardinality of the condition attribute positive domain: $\operatorname{Card}\left(P_{C}(D)\right), \operatorname{Card}\left(P_{C-\left\{c_{j}\right\}}(D)\right)$;

(4)Determine the dependency w of decision attribute set $D$ on condition attribute set $C: \gamma_{C}(D)$;

(5)Determine the dependency $\gamma_{C-\left\{c_{j}\right\}}(D)$ of decision attribute set $\mathrm{D}$ on each condition attribute $C-\left\{c_{j}\right\}$;

(6)Determine the importance degree $Z\left(c_{j}, C, D\right)$ of the condition attribute $c_{\mathrm{j}}$ in the condition attribute set $C$;

(7)Weighting the importance degree, and obtaining the weight coefficient of each condition attribute:

$$
\beta_{j}=\frac{Z\left(c_{j}, C, D\right)}{\sum_{j=1}^{n} Z\left(c_{j}, C, D\right)}
$$

(8)According to formula (6), formula (7) and each index score obtained by formula (8), the comprehensive score is:

$$
G=\sum_{j=1}^{n} \beta_{j} y_{i j}(i=1,2, \cdots, m)
$$

\section{CASE ANALYSIS}

Taking the coordination evaluation of a certain power network planning index as an example, it shows how to use the rough set to determine the index weight.

Proceed as follows: establishment of data decision table. According to a local power grid planning program, select the index data and establish the data decision table. Among them, the row is the evaluation object, for 6 years of data of 3 area, a total of 18 to be evaluated object, the column is selected to be evaluation index including: the highest harmonized load $\mathrm{c} 1$, power supply load of $220 \mathrm{kV}$ substation $\mathrm{c} 2,220 \mathrm{kV}$ capacity-to-load ratio c3, direct supply load of $220 \mathrm{kV}$ to $10 \mathrm{kV} \mathrm{c} 4,110 \mathrm{kV}$ capacity-to-load ratio c5, 110kV sensitive load capacity-to-load ratio $c 6$, the total electricity consumption c7, Public substation capacity c8. Condition attribute set $C=\left\{c_{1}, c_{2}, c_{3}, c_{4}, c_{5}, c_{6}, c_{7}, c_{8}\right\}$, decision attribute set $D=\{y\}$, y is the discrete value of the average score of each index.

As shown in Table 1, index for 6 years of 3 areas are listed, then the weight of each attribute is obtained by formula (8), and the weight coefficient value of each condition attribute is obtained.

\begin{tabular}{|c|c|c|c|c|c|c|c|c|c|}
\hline \multirow[b]{2}{*}{ area } & \multirow[b]{2}{*}{ year } & \multicolumn{8}{|c|}{ indexes $C$} \\
\hline & & $c_{1}$ & $c_{2}$ & $c_{3}$ & $c_{4}$ & $c_{5}$ & $c_{6}$ & $c_{7}$ & $c_{8}$ \\
\hline \multirow{6}{*}{1} & 2015 & 222 & 192 & 2.2 & 11 & 1.9 & 2 & 17.46 & 420 \\
\hline & 2016 & 234 & 204 & 2.1 & 14 & 1.8 & 1.9 & 18.82 & 420 \\
\hline & 2017 & 259 & 229 & 1.8 & 14 & 2 & 2.1 & 20.27 & 420 \\
\hline & 2018 & 284 & 254 & 2.4 & 15 & 1.9 & 2.1 & 21.84 & 600 \\
\hline & 2019 & 331 & 301 & 2 & 15 & 2 & 2.1 & 23.35 & 900 \\
\hline & 2020 & 366 & 336 & 2.7 & 20 & 1.9 & 2.1 & 25.54 & 900 \\
\hline \multirow[t]{8}{*}{2} & 2015 & 311 & 191 & 3.6 & 18 & 2.2 & 2.3 & 22.54 & 690 \\
\hline & 2016 & 339 & 219 & 3.2 & 19 & 2.1 & 2.2 & 24.28 & 690 \\
\hline & 2017 & 351 & 226 & 3.1 & 20 & 2.1 & 2.5 & 26.16 & 690 \\
\hline & 2018 & 396 & 271 & 2.5 & 21 & 1.9 & 2.2 & 28.18 & 690 \\
\hline & 2019 & 467 & 342 & 2 & 22 & 2 & 2.3 & 30.13 & 690 \\
\hline & 2020 & 494 & 364 & 1.9 & 24 & 1.8 & 2.1 & 32.95 & 690 \\
\hline & 2015 & 127 & 107 & 1.7 & 4 & 1.9 & 2 & 4.23 & 180 \\
\hline & 2016 & 152 & 132 & 1.4 & 6 & 1.5 & 1.6 & 4.56 & 180 \\
\hline \multirow[t]{4}{*}{3} & 2017 & 182 & 142 & 2.5 & 6 & 1.7 & 1.9 & 4.91 & 360 \\
\hline & 2018 & 199 & 159 & 2.3 & 7 & 2 & 2.2 & 5.29 & 360 \\
\hline & 2019 & 228 & 188 & 1.9 & 7 & 2.2 & 2.4 & 5.66 & 360 \\
\hline & 2020 & 241 & 201 & 1.8 & 8 & 2 & 2.2 & 6.19 & 360 \\
\hline
\end{tabular}

TABLE I. EVALUATION INDEXES 
TABLE II. EVALUATION GRADES

\begin{tabular}{lcccccccccc}
\hline \multirow{2}{*}{ area } & year & $y_{1}$ & $y_{2}$ & $y_{3}$ & $y_{4}$ & $y_{5}$ & $y_{6}$ & $y_{7}$ & $y_{8}$ & $D$ \\
\hline 1 & 2015 & 26 & 33 & 36 & 23 & 40 & 64 & 54 & 67 & 1 \\
& 2016 & 29 & 38 & 32 & 31 & 30 & 73 & 49 & 67 & 2 \\
& 2017 & 36 & 47 & 18 & 31 & 50 & 55 & 44 & 67 & 1 \\
& 2018 & 43 & 57 & 45 & 34 & 40 & 55 & 39 & 42 & 1 \\
& 2019 & 56 & 75 & 27 & 34 & 50 & 55 & 33 & 42 & 1 \\
& 2020 & 65 & 89 & 59 & 49 & 40 & 55 & 26 & 0 & 2 \\
\hline 2 & 2015 & 50 & 33 & 100 & 43 & 70 & 36 & 36 & 29 & 1 \\
& 2016 & 58 & 44 & 82 & 46 & 60 & 45 & 30 & 29 & 1 \\
& 2017 & 61 & 46 & 77 & 49 & 60 & 18 & 24 & 29 & 2 \\
& 2018 & 73 & 64 & 50 & 51 & 40 & 45 & 17 & 29 & 1 \\
& 2019 & 93 & 91 & 27 & 54 & 50 & 36 & 10 & 29 & 2 \\
& 2020 & 100 & 100 & 23 & 60 & 30 & 55 & 0 & 29 & 2 \\
\hline 3 & 2015 & 0 & 0 & 14 & 3 & 40 & 64 & 100 & 100 & 1 \\
& 2016 & 7 & 10 & 0 & 9 & 0 & 100 & 99 & 100 & 2 \\
& 2017 & 15 & 14 & 50 & 9 & 20 & 73 & 98 & 75 & 1 \\
& 2018 & 20 & 20 & 41 & 11 & 50 & 45 & 96 & 75 & 1 \\
& 2019 & 28 & 32 & 23 & 11 & 70 & 27 & 95 & 75 & 1 \\
& 2020 & 31 & 37 & 18 & 14 & 50 & 45 & 93 & 75 & 2 \\
\hline & & & & & & & & & &
\end{tabular}

Then the weight of each attribute is obtained by formula (8), and the weight coefficient value of each condition attribute is obtained:

$$
\begin{gathered}
\beta_{1}=0.1905, \beta_{2}=0.1429, \beta_{3}=0.1429, \beta_{4}=-0.0476, \beta_{5}=0.2587, \\
\beta_{6}=0.0476, \beta_{7}=0.1905, \beta_{8}=0.0476
\end{gathered}
$$

Take the first area in 2015 as an example, the scores of each index obtained by the formula (7) and the formula (8) are shown in Table 2, the comprehensive evaluation result can be calculated as 42 points by formula (11). A total of 18 evaluation object of 3 areas for 6 year, the minimum score is 31 and the maximum score is 62 . According to the equidistant method grading standards: poor (31 38.75), medium (38.75+ 46.5), excellent $(54.25+\sim 62)$, the first section of the grid planning coordination assessment in 2015 level of "medium". Through the comprehensive evaluation of each index, the level of area of each year network planning coordination assessment can be determined.

\section{CONCLUSION}

In this paper, an evaluation method based on rough set theory is used to evaluate the coordination of power network planning. The main idea of this paper is determining the weight coefficient of each evaluation indicator by the principle of attribute importance in rough set theory, reflecting the extent of the impact of the indexes in terms of coordination, which avoids the shortcomings of traditional evaluation methods in index empowerment. However, since the determination of the attribute weights of rough sets is completely dependent on the data, if the selected index data and the representation is not enough, the index weight obtained by analyzing the relationship between data will deviate from the actual, and does not match the actual degree of importance of the indexes in the index system. Therefore, in the use of the method, we should try to select a generally representative index data.

\section{ACKNOWLEDGMENT}

This work is supported by grant 2012BBE50049 of the Jiangxi Province Science and Technology Program Key Project.

\section{REFERENCES}

[1] Wei Liu, Power grid planning under electricity market, Guangdong Electric Power(Guangzhou, China, 1993), pp. 14-16.

[2] Ke-wen Wang and Mei-ping Fu, Research on evaluation of power network coordination and it's coordinated planning, Master's thesis, Zhengzhou University(2010).

[3] Hong-wei Chen, Qian $\mathrm{Xu}$ and Xiao Zheng, Integrated and coordinated evaluation of grid planning program based on combination weighting method, Zhejiang Electric Power(Hangzhou, China, 2014), pp. 1-5.

[4] Zhi-jie Song and Xiao-hong Gao, A method of index weighting setting in multi-criteria synthetical evaluation, Journal of Yanshan University(Yanshan, China,2002), pp. 20-22.

[5] Hui-min Liu, Topsis with synthetical weight for evaluation of logistics company performence, Logistics Technology(Xiangyang, China, 2009), pp. 95-97.

[6] Fu-hua Shu, Comprehensive power quality evaluation based on rough set, Electric Power Automation Equipment(Nanjing, China, 2008), pp. 75-79.

[7] Ming-xiao Liu, Study on attribute reduction based on rough sets and it's application, Master's thesis, Heibei University of Technology(2007)

[8] PAWLAK Z, Rough Set. International Journal of Computer Information Sciences, (1982), pp. 342-356.

[9] Jing Ma, Dong Xu and Zeng-ping Wang. Power restoration strategy based on weighted ideal point method for distribution network. Electric Power Automation Equipment(Nanjing, China, 2014), pp. 61 $-67$. 